\title{
Enterprise information risk management based on multi-agent model
}

\author{
Cao $\mathrm{Hu}^{1^{*}}$, Luo Haining ${ }^{2}$, Jiao $\mathrm{Di}^{2}$, Ren $\mathrm{Fei}^{2}$, Jiang Dongxing ${ }^{1}$, Tang Ming ${ }^{1}$ \\ ${ }^{1}$ Westone Information Industry Inc., Beijing 100067, China \\ ${ }^{2}$ State Information Center, Beijing 100045, China
}

Corresponding Author Email: job_caohu@163.com

https://doi.org/10.18280/rces.050303

Received: 27 April 2018

Accepted: 18 August 2018

\section{Keywords:}

risk management, enterprise information, multi-agent system, potential function

\begin{abstract}
This paper creates a risk management model of enterprise information based on graph theory and the multi-agent system, and discloses the relationship between agents and information elements. According to the OSI framework, five information elements were included in the modelling, namely, authentication, access control, data security, data integrity and nonrepudiation. The potential functions were introduced to depict the interaction between various information elements: the attractive force means the agents hold the same opinions, and the repulsive force means the agents disagree with each other, calling for adjustment. The control law based on smooth potential functions was also adopted for the modelling process. Finally, the proposed model was proved innovative and effective through a simulation on five robots. The research findings shed new light on risk management of enterprise information.
\end{abstract}

\section{INTRODUCTION}

Information is the result of the interconnection and interaction between various objective things. With such characteristics as objectivity, universality and transferability, information reflects the movement and state variation of the objective things. Information security directly bears on the profit and risk prevention of enterprises. To manage its information risks, an enterprise needs to evaluate the risks and adopt proper control measures at an acceptable cost.

According to the ISO7498-2, the risk management of enterprise information should include five security services available in the OSI reference model framework, namely, authentication, access control, data security, data integrity and non-repudiation. This calls for a human-machine system that supports specific applications by collecting and processing information. This system, also known as the information system, often consists of a computer and a number of relevant devices/facilities.

Risk analysis refers to the identification and estimation of risks in a project or event, that is, to identify all the factors that may generate risks, calculate the risk probability, predict the possible loss of a risk, and determine the risk level of a project or event $[1,2]$. Currently, the multi-agent system is a popular tool for risk analysis at home and abroad [3-5].

\section{MULTI-AGENT SYSTEM}

As its name suggest, a multi-agent system is composed of multiple agents. Compared to a single-agent system, the multiagent system can complete complex tasks, facilitate the design and production, and enhance the robustness and fault tolerance of the entire mechanism. Considering the interaction between agents, the multi-agent system is a linear superposition of multiple agents, rather than a simple combination of individual agents. In the system, the agents interact with each other in two ways, namely, coordination and cooperation. The multi-agent coordination and cooperation is mostly controlled by centralized methods or distributed methods. The centralized methods were developed earlier than the distributed methods, but the distributed methods are more popular nowadays, thanks to the fast development of distributed programming.

By centralized methods, the multi-agent system is deemed as a complex agent with multiple degrees of freedom (DOFs), and the interaction between individual agents is controlled according to a unified plan. The centralized methods can find the optimal solution without considering the complexity of calculation and programming. Nevertheless, much time is consumed to execute the algorithm in this type of methods.

By distributed methods, each agent collects information from its surroundings and the adjacent agents, and, on this basis, plans a relative optimal path towards the target. This type of methods enjoys high computing efficiency and fast running speed, and does not have to consider the condition of the global optimal solution. However, the distributed methods may lag behind their centralized counterparts in runtime and even fail to reach the optimal solution.

\section{ENTERPRISE INFORMATION FRAMEWORK BASED ON GRAPH THEORY}

The conclusions and techniques of the graph theory have been widely adopted to study the multi-agent system. The multi-agent system can be described accurately using the language and symbols of the graph theory, as the system covers undirected, directed and weighted connected networks.

According to the graph theory [6], an undirected graph can be expressed as $\mathrm{G}=(\mathrm{V}, \mathrm{E})$, with $V=\left(v_{1}, v_{2}, \ldots, v_{n}\right) \in R^{2 n}$ and $E=\left(e_{1}\right.$, $\left.e_{2}, \ldots, e_{n}\right)$ being $\mathrm{n}$ vertices and $\mathrm{m}$ edges, respectively. Let $|\mathrm{V}|$ be the number of vertices and $|\mathrm{E}|$ be the number of edges. Assuming that each vertex stands for an agent, the undirected graph $|\mathrm{E}|=2|\mathrm{~V}|-3$ can achieve a stable state based on the 
principle of multi-agent rigid formation. The stable undirected graph is presented in Figure 1 below, in which $|V|=5$ and $|E|=7$.

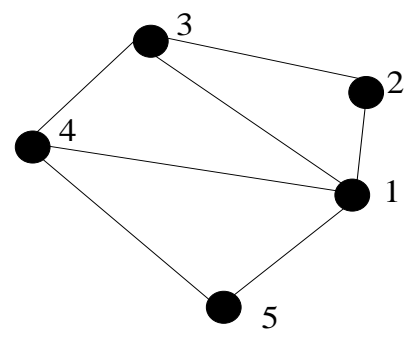

Figure 1. Stable undirected graph

As mentioned before, the information of an enterprise usually covers the following five areas: Agent 1 identity authentication, Agent 2 access control, Agent 3 data security, Agent 4 data integrity and Agent 5 non-repudiation.

\section{MULTI-AGENT INFORMATION MODEL}

The risk management of enterprise information aims to transform each agent from a disordered state to an ordered and operable state. Inspired by References [1, 2], the author adopted the potential functions between the multiple agents for the modelling of multi-agent information of enterprises. With the potential functions, a repulsive force exists between two agents when the distance between them is below a pre-set threshold, and an attractive force exists between them when the distance is greater than that threshold. The forces keep changing until reaching a stable operating state.

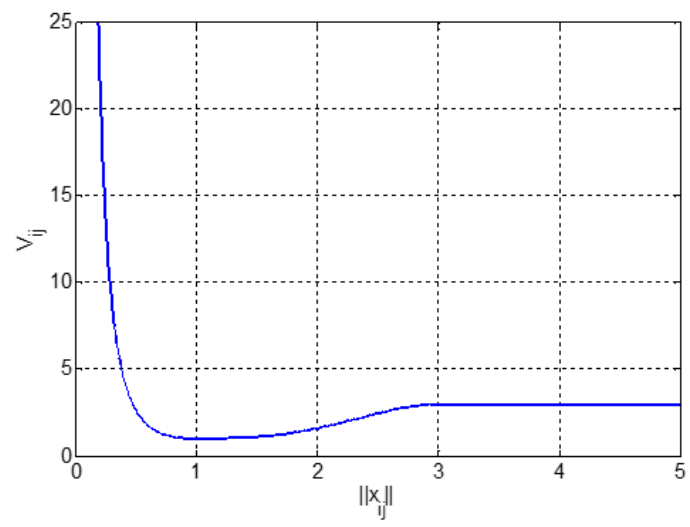

Figure 2. The potential function between agent $i$ and agent $j$

Here, the potential functions are considered as the constraint on enterprise information, under which all departments and systems can reach a stable operating condition. In our multiagent information model, the smooth potential functions are adopted:

$$
V_{i j}\left(\left\|x_{i j}\right\|\right)=\left\{\begin{array}{l}
a \ln \left\|x_{i j}\right\|^{2}+\frac{b}{\left\|x_{i j}\right\|^{2}} \quad 0<\left\|x_{i j}\right\| \leq \sqrt{\frac{b}{a}} \\
a \ln \left\|x_{i j}\right\|^{2}+\frac{b}{\left\|x_{i j}\right\|^{2}}+\cos \left(1+\frac{\left\|x_{i j}\right\|^{2}-\frac{b}{a}}{R^{2}-\frac{b}{a}}\right) \pi+1 \quad \sqrt{\frac{b}{a}}<\left\|x_{i j}\right\|<R_{d} \\
a \ln \left\|x_{i j}\right\|^{2}+\frac{b}{\left\|x_{i j}\right\|^{2}}+2 \text { otherwise }
\end{array}\right.
$$

where $a, b$ and $R_{d}$ are positive constants $\left(b>\frac{e}{a}, R_{d}>\sqrt{\frac{b}{a}}\right)$; $V_{i j}$ is the potential function between agent $i$ and agent $j ; x_{i j}$ is the distance between agent $i$ and agent $j$. As shown in Figure 2 below, $V_{i j}$ is an everywhere continuously differentiable function.

The potential function $V_{i}$ of agent $i$ can be derived in light of the features of $V_{i j}$ :

$$
V_{i}=\sum_{j \notin N_{i}} V_{i j}(R)+\sum_{j \in N_{i}} V_{i j}\left(\left\|x_{i j}\right\|\right)
$$

Then, the control law $u_{i}$ of agent $i$ can be expressas:

$u_{i}=-\sum_{j \in N_{i}} \nabla_{x_{i}} V_{i j}-\sum_{j \in N_{i}} w_{i j}\left(v_{i}-v_{j}\right)$

where $w_{i j}$ is the weight coefficient of each edge; the value of $w_{i j}$ is positively correlated with the tightness of the contact between agents in the multi-agent information model.

\section{EXPERIMENTAL VERIFICATION}

This section attempts to verify the feasibility of the proposed multi-agent information model through robotic simulation. As shown in Figure 3, five robots were selected as samples for the experimental verification. Each robot represent one of the five elements of enterprise information.

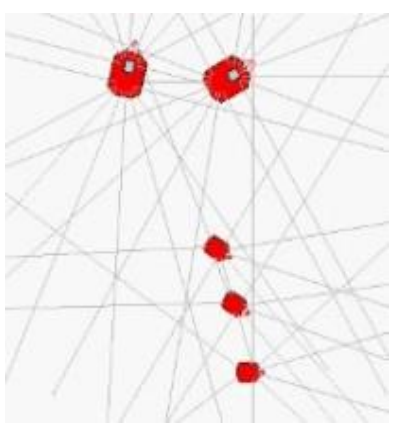

(a)

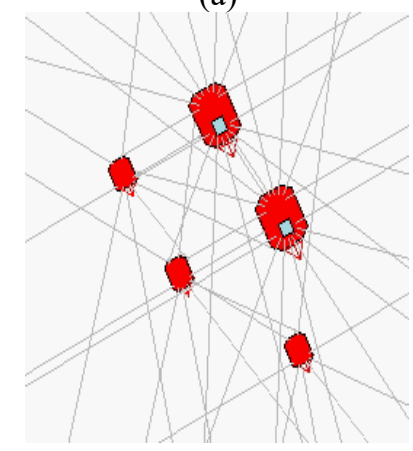

(c)

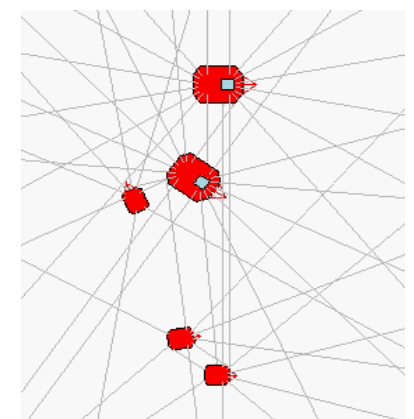

(b)

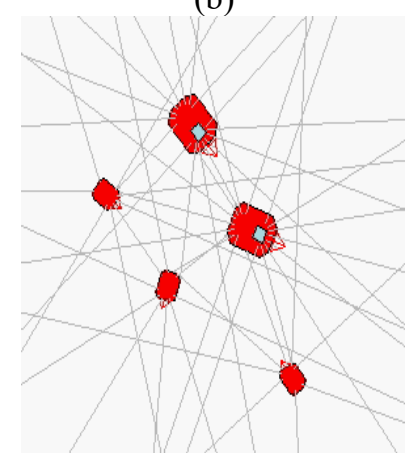

(d)
Figure 3. Robotic simulation

It can be seen that the robots are in the disordered state in Figure 3(a), while those in Figures 3(b) (d) have entered the ordered state under the action of the potential functions. This means the enterprise information has reached a stable state under the constraints. 


\section{CONCLUSIONS}

This paper creates a risk management model of enterprise information based on graph theory and the multi-agent system, and discloses the relationship between agents and information elements. The potential functions were introduced to depict the interaction between various information elements: the attractive force means the agents hold the same opinions, and the repulsive force means the agents disagree with each other, calling for adjustment. The control law based on smooth potential functions was also adopted for the modelling process. Finally, the proposed model was proved innovative and effective through a simulation on five robots. The research findings shed new light on risk management of enterprise information.

\section{REFERENCES}

[1] Agrawal V. (2017). A comparative study on information security risk analysis methods. Journal of Computers 12(1): 57-67. http://dx.doi.org/10.17706/jcp.12.1.57-67
[2] Vlek C, Stallen PJ. (1981). Judging risks and benefits in the small and in the large enterprise. Organizational Behavior and Human Performance 28(2): 235-271. http://dx.doi.org/10.1016/0030-5073(81)90024-6

[3] Cao H, Bai Y, Liu H. (2012). Distributed rigid formation control algorithm for multi-agent systems. Kybernetes 41(10): $1650-1661$ http://dx.doi.org/10.1108/03684921211276819

[4] Cao H, Bai Y, Chen J, Fang H. (2013). Control of 2D minimally persistent formations with three co-leaders in a cycle. International Journal of Advanced Robotic Systems 10(21): 1-7. http://dx.doi.org/10.5772/54494

[5] Olfati-Saber R, Murray R. (2002). Distributed cooperative control of multiple vehicle formations using structural potential functions. IFAC Proceedings Volumes $35(1)$ : 495-500. http://dx.doi.org/10.3182/20020721-6-ES-1901.00244

[6] Asimow L, Roth B. (1978). Rigidity of graphs. Transactions of the American Mathematical Society (245): 279-289. http://dx.doi.org/10.1090/S0002-99471978-0511410-9 